\title{
Recovery of menstruation and pregnancy after liver transplantation
}

\author{
T F Cundy, ${ }^{\star}$ J G O’Grady, $†$, Roger Williams
}

\begin{abstract}
The effect of successful liver transplantation on menstrual function was assessed by questionnaire in $\mathbf{4 4}$ women transplanted for various types of end stage liver disease, acute liver failure or malignant disease. Significant amenorrhoea (>one year) was present in $48 \%$ of women with chronic liver disease before transplantation, and was reversed within 10 months of surgery in all but one of the premenopausal patients who had primary amenorrhoea and hypogonadotrophic hypogonadism. Two patients became pregnant five months and 1.5 years after transplantation, but in one instance the pregnancy was unsuccessful, possibly as a consequence of cyclosporin related intrauterine growth retardation.
\end{abstract}

Amenorrhoea is a recognised complication of all types of end stage chronic liver disease. In some cases of autoimmune chronic active hepatitis, amenorrhoea occurs relatively early in the disease process, while in primary biliary cirrhosis menorrhagia is the predominant initial menstrual abnormality.' Successful pregnancy has been documented in patients with autoimmune chronic active hepatitis who are well controlled with immunosuppressive therapy, usually prednisolone and azathioprine. ${ }^{23}$ The potential for reversibility of menstrual disturbance in more advanced cases is uncertain, however, and consequently we have studied the effect of successful liver transplantation on menstrual function. This is also of clinical importance, as with the rapid expansion of transplantation programmes there will be an increasing number of women so treated seeking advice on fertility. Some who have completed their families may require advice on the need for contraception, while others will be anxious for guidance on the possibility of becoming pregnant.

Department of Medicine and Liver Unit, $†$ King's College Hospital, and School of Medicine and Dentistry, London T F Cundy J G O'Grady Roger Williams Correspondence to Dr Roger Williams, Director of Liver Unit, King's College London SE5 8RX.

Accepted for publication 23 May 1989
A postal questionnaire was sent to all 44 women between the ages of 17 and 54 years who underwent orthotopic liver transplantation in the Cambridge/King's College Hospital joint programme between 1976 and 1987 and who were alive on 31 March, 1987. The patients were divided into three subgroups on the basis of aetiology: (a) 16 patients with primary biliary cirrhosis (PBC); (b) 16 patients with other forms of end stage liver disease (eight chronic active hepatitis, three Budd-Chiari syndrome, two sclerosing cholangitis, one posthepatitic cirrhosis, one alcoholic cirrhosis, one Wilson's disease); (c) seven patients without chronic liver disease (five acute liver failure, two malignancy). All but two of the patients were taking cyclosporin for maintenance immunosuppression and all had good graft function.

The information sought on the questionnaire included details of menarche, hormonal contraceptive use, gynaecological procedures and menstrual pattern before and after transplantation. The endocrine status of six patients who remained amenorrhoeic post-transplantation was subsequently assessed by measuring serum gonadotrophin concentrations. The Wilcoxon's T-test was used for statistical analysis.

\section{Results}

Replies were received from 39 (89\%) of the 44 patients, with four of the five non-responders being resident outside Great Britain. Five women with chronic liver disease (four group (a), one group (b)) had undergone hysterectomy or radiation menopause for menorrhagia between one and 10 years before transplantation. Amenorhoea of >one year was reported in $48 \%$ of women with chronic liver disease (groups (a) and (b)) but in none of the patients in group (c). There were no differences in the frequency or duration of amenorrhoea between groups (a) and (b) (Table I). Of the 34 patients potentially able to resume menstruation, 28 did so within one and 10 months of liver transplantation. Five of the remaining six patients (aged 48-54 years) had greatly raised serum gonadotrophin concentrations (FSH and LH both $>90 \mathrm{U} / \mathrm{l}$ ) indicating they were postmenopausal.

Among the 29. premenopausal women, menstruation had occurred by two months in 12 (41\%), by five months in $23(79 \%)$ and by seven months of transplantation in $26(90 \%)$ (Figure). The interval between transplantation and resumption of menstruation did not differ between the three subgroups. Remarkably, two women with primary biliary cirrhosis resumed menstruating at the ages of 51 and 54 years after amenorrheic periods of 1.5 and five years, respectively. The only patient not to start menstruating within one year of transplantation was 
TABLE Findings relating to return of menstruation after liver transplantation in the three clinical subgroups of patients

\begin{tabular}{llll}
\hline Group $(a)$ & $\begin{array}{l}\text { Group }(b) \\
n=16\end{array}$ & $\begin{array}{l}\text { Group }(c) \\
n=16\end{array}$ & $n=7$ \\
\hline Previous hysterectomy or radiation menopause & 4 & 1 & 0 \\
Postmenopausal & 3 & 1 & 1 \\
Number with potential to resume menstruation & 9 & 14 & 6 \\
Age (range) yr & $46(35-54)$ & $30(17-49)$ & $28(22-42)$ \\
Median duration of amenorrhoea before & $0 \cdot 4(0-5)$ & $1 \cdot 3(0-17)$ & $0 \cdot 3(0-0 \cdot 8)$ \\
$\quad$ transplant (range) yr & $3(33 \%)$ & $8(57 \%)$ & 0 \\
$\quad \rightarrow$ l year & 9 & 13 & 6 \\
Return of menstruation & $3(1-10)$ & $3(1-10) \dagger$ & $2(1-6)$ \\
Duration of amenorrhoea post-transplantation (mo) & & & \\
\hline
\end{tabular}

*Age $>47$ yr and raised serum gonadotrophins.

†Including one patient with primary amenorrhoea.

aged 28 years, had autoimmune chronic active hepatitis since the age of six years and primary amenorrhoea. Investigations at 10 months posttransplantation showed the presence of hypogonadotrophic hypogonadism (LH 3.1 U/1, FSH $4 \mathrm{U} / \mathrm{l}$ ), with a very low serum oestradiol concentration and multicystic ovaries on ultrasonic examination.

There have been two pregnancies in this cohort. The first, a 25 year old patient, conceived 15 months after liver transplantation for posthepatitic cirrhosis. Maintenance immunosuppression during pregnancy was with cyclosporin $100 \mathrm{mg}$ (cyclosporin levels $91-93 \mathrm{ng} / \mathrm{ml}$ in whole blood and measured by monoclonal radioimmunoassay), prednisolone $12.5 \mathrm{mg}$ and azathioprine $75 \mathrm{mg}$ daily. The pregnancy was not complicated by hypertension. Placental blood flow was found to be markedly reduced at six months and death in utero followed shortly afterwards. The second patient had amenorrhoea for nine years before undergoing liver transplantation for autoimmune chronic active hepatitis. She resumed menstruation three months post-transplantation, conceived twins at five months, and at 34 weeks delivered healthy twins after an uneventful pregnancy. This patient was intolerant of cyclosporin (nausea and headaches) and maintenance immunosuppression is with prednisolone $17.5 \mathrm{mg}$ and azathioprine $100 \mathrm{mg}$ daily. In both cases, liver function has remained normal during pregnancy.

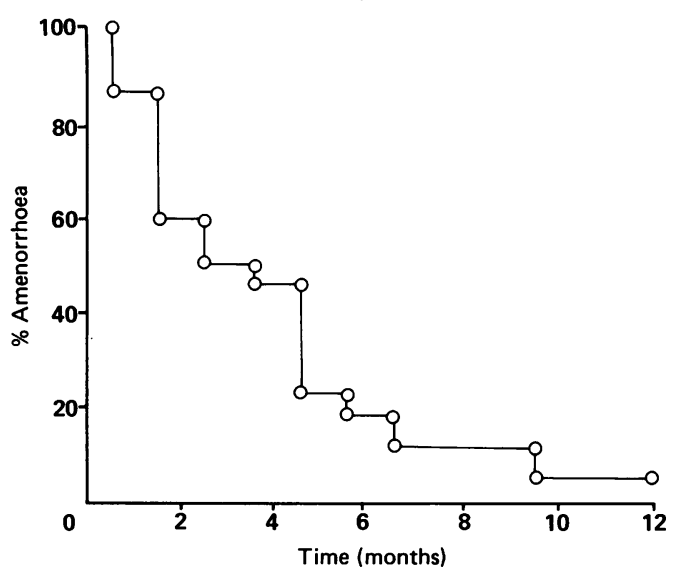

Figure: Percentage of patients with persistent amenorrhoea during the first year after liver transplantation.

\section{Discussion}

Significant amenorrhoea is a common clinical feature in women with chronic liver disease that has advanced to the stage where liver transplantation is needed. The prevalence of amenorrhoea appears to be somewhat higher in patients with cirrhosis and advanced hepatocellular failure than in those with biliary disease and predominant cholestasis. The latter are more likely to have undergone hysterectomy or radiation menopause for dysfunctional bleeding, as previously described. ${ }^{4}$ The hormonal basis for the amenorrhoea is poorly understood, but probably involves hypothalamic-pituitary dysfunction. This study indicates that the underlying defect is corrected quite quickly, and all premenopausal patients with secondary amenorrhoea had resumed menstruation within 10 months of transplantation. The interval between transplantation and resumption of menstruation did not correlate with age, duration of amenorrhoea or diagnosis. The resumption of regular menstruation in some women over the age of 50 years was unexpected. It might suggest that the normal age related decline in ovarian function might be interrupted during the period of gonadotrophin insufficiency caused by the advanced liver disease. The majority of patients with secondary amenorrhoea are likely to resume menstruation and endogenous oestrogen production, and those not wishing to conceive should use contraception from the time of resuming sexual intercourse. The oral contraceptive pill is not advised in patients with the Budd-Chiari syndrome, but should be considered in other cases. The need for oestrogen replacement therapy should be evaluated in those failing to menstruate within one year of transplantation.

Two successful pregnancies have previously been reported after liver transplantation before the introduction of cyclosporin. ${ }^{4}$ While there have been no published updates on pregnancy after liver transplantation, a recent report has suggested cyclosporin may have a deleterious effect during pregnancy after renal transplantation. ${ }^{5}$ In that series, nine of 16 infants showed evidence of growth retardation. This is similar to one of our patients whose infant died with growth retardation associated with decreased placental blood flow, presumably as a consequence of cyclosporin induced vasoconstriction, despite the low dose of cyclosporin used. Consequently, it is now our policy to maintain immunosuppression with prednisolone and azathioprine in future pregnancies. The successful twin pregnancy is the first multiple birth reported after orthotopic liver transplantation.

1 Powell D. Pregnancy in active chronic hepatitis on immunosuppressive therapy. Postgrad Med f 1969; 45: 292-4.

Steven MM, Buckley JD, Mackay IR. Pregnancy in chronic active hepatitis. $Q \mathcal{F}$ Med 1979; 192: 519-31.

3 Stellon AJ, Williams R. Increased incidence of menstrual abnormalities and hysterectomy preceding primary biliary cirrhosis. Br Med f 1986; 293: 297-8.

4 Penn I, Makowski EL, Harris P. Parenthood following renal and hepatic transplantation. Transplantation 1980; 30: 397400.

5 Pickrell MD, Sawers R, Michael J. Pregnancy after renal transplantation: severe intrauterine growth retardation during treatment with cyclosporine A. Br Med $\mathcal{F} 1988$; 296: 825 . 